\title{
Análise do desempenho do Índice de Desenvolvimento da Educação Básica (IDEB) de acordo com o custo das atividades relevantes
}

\author{
Leandro Saraiva Dantas de Oliveira \\ Mestrado em Ciências Contábeis pelo Multi UnB/UFPB/UFRN \\ Professor da Universidade Federal do Cariri - UFCA \\ E-mail: leandro.saraiva@hotmail.com
}

Victor Godeiro de Medeiros Lima Doutorado em andamento em Ciências Contábeis pela Universidade de Brasília - UnB E-mail:victor_godeiro@hotmail.com

Agamenon da Costa Melo Segundo Mestrado em Ciências Contábeis pelo Multi UnB/UFPB/UFRN E-mail: agamenonm@yahoo.com.br

Aneide Oliveira Araujo Doutorado em Controladoria e Contabilidade pela Universidade de São Paulo - USP Professora da Universidade Federal do Rio Grande do Norte - UFRN E-mail: aneide.ufrn@gmail.com

\section{RESUMO}

Buscou-se mensurar e avaliar as atividades relevantes para obtenção dos melhores desempenhos no IDEB nas últimas três Provas Brasil, em três escolas municipais da cidade de Natal. É um estudo qualitativo realizado por meio de análise do conteúdo das informações obtidas em entrevistas semiestruturadas com os diretores das escolas e documentos para cálculo dos custos utilizando uma adaptação do custeio por atividades. Foi apontada como diferencial, basicamente, a realização de atividades de leitura, simulados para capacitar os alunos e acompanhamento pedagógico. Logo, calcularam-se os gastos dessas atividades que agregam valor. A atividade que apresentou menor custo foi a Roda de Leitura, estimada em $R \$ 438,00$, valor esse próximo do alcançado pelas atividades de Simulado nas duas escolas, que teve os simulados como principal motivo para o sucesso das escolas, sendo eles de $R \$ 466,70$ e 619,01. Já a atividade com maior valor de custos é o Acompanhamento Pedagógico Semanal, estimado em $R \$ 102.507,80$. A mensuração dos custos das atividades sugeridas como diferenciais proporciona mecanismos de avaliação de resultados, permitindo a comparação dos custos entre escolas municipais e um melhor gerenciamento dos recursos ao alocá-los em atividades pedagógicas diferenciais com o objetivo de melhorar seu desempenho.

Palavras-chave: Custo no setor público. Educação básica. Gasto público. 
Análise do desempenho no Índice de Desenvolvimento da Educação Básica (IDEB) de acordo com o custo das atividades relevantes

Leandro Saraiva Dantas de Oliveira, Victor Godeiro de Medeiros Lima, Agamenon da Costa Melo

Segundo, Aneide Oliveira Araujo

Performance analysis in the Basic Education Development Index (IDEB) according to the cost of the relevant activities

\section{ABSTRACT}

It was sought to measure and evaluate the relevant activities to obtain the best performances in the IDEB, in the last three brazilian tests in three municipal schools in the city of Natal. It is a qualitative study carried out by analyzing the content of the information obtained in semistructured interviews with school principals and documents for calculating costs using an adaptation of activity-based costing. It was pointed out as a differential, basically, the performance of reading activities, simulated to train the students and pedagogical accompaniment. Therefore, the expenses of these activities that add value were calculated. The activity that presented the lowest cost was the Reading Wheel, estimated at $R \$ 438.00$, an amount close to that achieved by the Simulated activities in the two schools that had the simulated as the main reason for the success of the schools, being $R \$ 466.70$ and 619.01 . The activity with the greatest cost value is the Weekly Pedagogical Accompaniment, estimated at $R \$ 102,507.80$. Measuring the costs of activities suggested as differentials provides mechanisms for evaluating results, allowing the comparison of costs between municipal schools and a better management of resources by allocating them in differential pedagogical activities in order to improve their performance.

Keywords: Costs in the public sector. Basic education. Public expenditure.

\section{INTRODUÇÃO}

Medir os custos de um determinado serviço é importante para as tomadas de decisões sobre a sua viabilidade, principalmente quando comparado com os resultados alcançados. O setor público brasileiro tem despertado para os benefícios dessa importante ferramenta de gestão com o propósito de otimizar os recursos públicos e assim oferecer melhores serviços.

Uma das mais importantes atribuições da administração pública consiste na promoção de uma educação de qualidade, visando à formação de seus cidadãos e ao combate à desigualdade social, contribuindo para o desenvolvimento do país.

Nesse sentido, Meyer, Ramirez, Rubinson e Boli-Bennett (1977) verificaram que se tornou universalmente aceito, no século $X X$, que a educação é um dos pilares 
Análise do desempenho no Índice de Desenvolvimento da Educação Básica (IDEB) de acordo com o custo das atividades relevantes

Leandro Saraiva Dantas de Oliveira, Victor Godeiro de Medeiros Lima, Agamenon da Costa Melo

Segundo, Aneide Oliveira Araujo

básicos para se chegar a uma sociedade igualitária. Isso resultou em grande expansão do setor educacional, principalmente a educação pública. Na mesma linha de pensamento, Soubbotina (2004) entende que os governos têm investido mais recursos públicos na educação, pois uma população capacitada contribuiria para um desenvolvimento sustentável do país de forma mais eficaz.

No Brasil, os estados e municípios têm a incumbência de prover a educação básica para a população, com significativas variações regionais e locais nessas responsabilizações (Farenzena \& Machado, 2006). Um dos métodos de avaliação do desempenho deste nível de educação é o Índice de Desenvolvimento da Educação Básica (IDEB). Este indicador sintetiza dois conceitos importantes para a qualidade da educação, que são: aprovação e média de desempenho dos estudantes em língua portuguesa e matemática. É calculado a partir dos dados sobre aprovação escolar e médias de desempenho nas avaliações do Instituto Nacional de Estudos e Pesquisas Educacionais Anísio Teixeira (INEP), chamados de Sistema de Avaliação da Educação Básica (SAEB) e a Prova Brasil.

Conforme observado no sítio do INEP, essas avaliações são aplicadas apenas nas $5 .^{\mathrm{a}}$ e $9 .^{\mathrm{a}}$ séries para verificar 0 aprendizado das séries iniciais e finais, respectivamente, do Ensino Fundamental. O resultado nacional dos anos iniciais, para o exame realizado em 2011, foi nota 5. O Rio Grande do Norte apresentou um desempenho inferior à média nacional, com a nota 4,1 . Analisando as escolas da rede pública estadual, o resultado foi ainda pior, com a nota 3,7, o terceiro pior do país. As escolas da rede pública municipal da cidade de Natal/RN apresentaram o resultado 4,0, um pouco melhor que a média estadual, mas ainda aquém da média nacional. (Assessoria de Imprensa do Inep/MEC, 2011).

Entretanto, entre esses resultados alcançados, existem escolas públicas municipais na cidade de Natal que obtiveram índices acima da média nacional, sendo necessário conhecer e medir os custos das atividades que foram desenvolvidas, nesses casos, que contribuíram para alcançar esses melhores desempenhos para que sirvam de benchmarking para as demais escolas públicas norte-rio-grandenses. 
Análise do desempenho no Índice de Desenvolvimento da Educação Básica (IDEB) de acordo com o custo das atividades relevantes

Leandro Saraiva Dantas de Oliveira, Victor Godeiro de Medeiros Lima, Agamenon da Costa Melo

Segundo, Aneide Oliveira Araujo

Duncombe, Ruggiero e Yinger (1995) entendem que, em nível conceitual, a importância de se determinar o custo educacional não pode ser negada. Diante desses fatos, o presente estudo tem a seguinte problemática de pesquisa: Quais os custos das atividades que agregam valor para o desempenho escolar no IDEB? O objetivo geral desse estudo é mensurar os custos das atividades consideradas importantes para o bom desempenho no IDEB das melhores escolas públicas da rede municipal da cidade de Natal. Para tanto, define-se como objetivos específicos: (i) identificar as atividades relevantes para o melhor desempenho; (ii) Conhecer os recursos alocados em cada escola; (iii) Determinar os custos de cada atividade relevante desenvolvida.

Portanto, este trabalho se justifica, pois, apesar de estudos recentes terem buscado identificar relações existentes entre os gastos públicos gerais em educação e o desempenho no IDEB (Adriano, Rosa, \& Flach, 2015; Borinelli, Amâncio-Vieira, Negreiros, Pereira, \& Pereira, 2014; Crozatti \& Almeida, 2012; Diniz \& Corrar, 2011), percebe-se uma escassez de produção científica relacionada especificamente a identificar quais atividades desenvolvidas nas escolas foram fatores determinantes para melhorar seus desempenhos e quais custos estão relacionados a essas atividades.

Logo, espera-se que outras escolas municipais possam utilizar as técnicas adotadas pelas escolas analisadas no presente estudo, como benchmarking, aplicando algumas das atividades destacadas com o intuito de melhorar o desempenho de seus alunos, gerando, possivelmente, impactos positivos na educação dos discentes e trazendo maior retorno à sociedade.

Para cumprir esses objetivos, o artigo foi estruturado em cinco seções: esta introdução onde se contextualiza e se define a questão que orienta a presente pesquisa, bem como os objetivos a serem atingidos; na próxima, apresenta-se a revisão da literatura acerca da evolução, objetivos e elementos dos custos no setor público, bem como do custeio baseado em atividades; na seção três, consta a descrição da metodologia empregada neste estudo; na seção quatro, descreve-se a análise de dados da pesquisa; e a última seção compreende as conclusões sobre o estudo realizado e indicações para futuras pesquisas; por fim, as referências utilizadas. 
Análise do desempenho no Índice de Desenvolvimento da Educação Básica (IDEB) de acordo com o custo das atividades relevantes Leandro Saraiva Dantas de Oliveira, Victor Godeiro de Medeiros Lima, Agamenon da Costa Melo Segundo, Aneide Oliveira Araujo

\section{FUNDAMENTAÇÃO TEÓRICA}

Este tópico apresenta a fundamentação teórica do estudo, sendo composto, inicialmente, do contexto histórico e consequente evolução da contabilidade de custos aplicada ao setor público, discutindo o embasamento legal e doutrinário, até apresentar o custeio baseado em atividades como uma possível ferramenta para a contabilidade pública.

\subsection{Evolução Histórica}

A implementação da contabilidade de custos para as entidades públicas brasileiras é recente, apesar de se encontrarem ditames legais desde meados da década de 1960 (Cardoso, Aquino, \& Bitti, 2011). No entanto, a Lei 4.320/64, quando aborda a implantação de um sistema de custos, trata, tão somente, dos serviços industriais por meio de seus artigos 85 e 99. A obrigatoriedade foi ampliada pela Lei $101 / 2000$, por meio de seus artigos $4 .$. e 50 , tornando-se uma ferramenta de uso para toda a contabilidade.

Mesmo com essas determinações legais, até o ano 2010, era raro o órgão público que se utilizava de informações de custos, porque, até então, não havia nem sistema, nem metodologia adequada capaz de ser aplicada, sistemática e uniformemente, para toda a administração pública federal (Machado \& Holanda, 2010).

A discussão sobre a adoção de custos no setor público só tomou força após a convergência às normas internacionais. Esse processo teve início, oficialmente, a partir da publicação, pelo Ministério da Fazenda, da Portaria no 18/2008 e, também, no mesmo ano, com a emissão, pelo Conselho Federal de Contabilidade, das primeiras Normas Brasileiras de Contabilidade Aplicadas ao Setor Público (NBCASP) (Cruvinel \& Lima, 2011).

Nesse sentido, por meio da Norma Brasileira de Contabilidade Técnica (NBC T) 16.2, aprovada pela Resolução CFC № 1.129/08, houve mudanças nos subsistemas contábeis que representam a estrutura de informações sobre identificação, 
Análise do desempenho no Índice de Desenvolvimento da Educação Básica (IDEB) de acordo com o custo das atividades relevantes Leandro Saraiva Dantas de Oliveira, Victor Godeiro de Medeiros Lima, Agamenon da Costa Melo Segundo, Aneide Oliveira Araujo

mensuração, avaliação, registro, controle e evidenciação dos atos e dos fatos da gestão do patrimônio público. O objetivo foi orientar e suprir o processo de decisão, a prestação de contas e a instrumentalização do controle social, onde o subsistema financeiro foi incorporado pelo subsistema patrimonial, instituindo-se o de custos com o objetivo de registrar, processar e evidenciar os custos dos bens e serviços produzidos e ofertados à sociedade pela entidade pública. (NCBT 16.2).

Entretanto, somente por meio da Resolução CFC № 1.366/11 foi dado um melhor detalhamento do Sistema de Informações de Custos do Setor Público (SICSP), bem como destaque para o valor dessa informação para fins gerenciais, além dos aspectos legais.

Dessa forma, o uso de um sistema de informações de custos será capaz de auxiliar os gestores públicos em suas decisões, como: ofertar ou terceirizar um serviço, produzir ou comprar um bem, ou ainda, comparar os custos de um mesmo serviço oferecido por órgãos diferentes e seus respectivos resultados, estimulando a melhoria do desempenho organizacional (Machado \& Holanda, 2010), principalmente em um momento da transformação da administração pública brasileira, que vem tentando dar foco maior aos resultados, à ampliação da transparência das ações governamentais, assim como à identificação de novas formas de prover os serviços públicos (Rezende, Cunha, \& Cardoso, 2010).

\subsection{Objetivos dos Custos no Setor Público}

O item 3 da Resolução CFC № 1.366/11, que aprovou a NBCT 16.11, cita os seguintes objetivos para o SICSP de bens e serviços: 
Análise do desempenho no Índice de Desenvolvimento da Educação Básica (IDEB) de acordo com o custo das atividades relevantes Leandro Saraiva Dantas de Oliveira, Victor Godeiro de Medeiros Lima, Agamenon da Costa Melo Segundo, Aneide Oliveira Araujo

$[\ldots]$

(b) apoiar a avaliação de resultados e desempenhos, permitindo a comparação entre os custos da entidade com os custos de outras entidades públicas, estimulando a melhoria do desempenho dessas entidades;

$[\ldots]$

(e) apoiar programas de controle de custos e de melhoria da qualidade do gasto.

Desse modo, a informação de custos no setor público pode ser usada como ferramenta de comparação da gestão de diferentes instituições, servindo como forma de mensurar a eficiência do gasto público, bem como a análise de implantação de determinada atividade de sucesso em outras instituições.

Segundo Machado e Holanda (2010), um dos principais objetivos do sistema de custos do governo federal é:

Maior eficiência operacional - a produção de bens e serviços pelo Estado deve se submeter a algumas avaliações, quanto às maneiras diferentes de produzir o mesmo produto com custos menores, que permitam identificar ineficiências operacionais e oportunidades de redução de gastos; quanto à avaliação do desempenho das pessoas e organizações (inclusive outros entes e ONGs); quanto à identificação de economias com despesas correntes (CF, art. 39, § 7. $\left.{ }^{\circ}\right)$ e também quanto ao custo/benefício das ações. Assim, facilita a identificação de ineficiências e oportunidades de redução de gastos; avaliação de resultado e desempenho, bem como benchmarking e identificação de atividades de baixo valor agregado.

Assim, o conhecimento dos custos das atividades pelo gestor é importante, pois poderá avaliar como eles estão sendo incorridos, e assim eliminar ineficiências, oferecendo serviços de melhor qualidade com custos menores.

Neste mesmo sentido, Cardoso, Aquino e Bitti (2011) citam que, nas entidades públicas, a utilidade da informação está associada ao uso que os gestores fazem dela, 
Análise do desempenho no Índice de Desenvolvimento da Educação Básica (IDEB) de acordo com o custo das atividades relevantes

Leandro Saraiva Dantas de Oliveira, Victor Godeiro de Medeiros Lima, Agamenon da Costa Melo

Segundo, Aneide Oliveira Araujo

dentre os quais: gerenciar atividades ou programas, atribuir preço ou taxa, análise de fornecer ou terceirizar atividades, avaliar o resultado de um programa, entre outros.

A utilização de informações de custos no setor público será de fundamental importância para a implantação de estratégias visando ao uso racional dos recursos públicos. Com essas informações, o gestor saberá analisar o custo/benefício de cada serviço oferecido, mediante a aferição da relação entre custos e resultado (Rezende, Cunha, \& Cardoso, 2010).

\subsection{Elementos do Sistema de Custos no Setor Público}

Segundo a NBC T 16.11, aprovada pela Resolução CFC № 1.366/11, o SICSP é apoiado em três elementos: sistema de acumulação; sistema de custeio e método de custeio. Na referida norma, é apresentado que o sistema de acumulação é a forma à qual os custos são acumulados e alocados aos bens e serviços, estando relacionados ao fluxo físico e real da produção.

Serviços públicos que são prestados de forma contínua, como ensino, assistência médica, distribuição da justiça, cuidado com a segurança pública e a defesa nacional, entre outros, requerem um sistema de acumulação de custos por processo. (Machado \& Holanda, 2010).

O sistema de custeio está vinculado ao modelo de mensuração, podendo os agentes de acumulação ser custeados de acordo com diferentes unidades de medidas, de acordo com as necessidades. No âmbito do sistema de custeio, podem ser utilizadas as seguintes unidades de medida: custo histórico; custo-corrente; custo estimado e custo padrão (NBCT 16.11).

Já o método de custeio se refere ao modo de apropriação de custos e está associado ao processo de identificação e associação do custo ao objeto que está sendo custeado. A escolha do método de custeio do setor público não é tarefa fácil, na medida em que há defensores para quase todos os métodos. Além disso, no setor público, diferentemente do setor privado, não há limitação legal quanto ao uso dos métodos de 
Análise do desempenho no Índice de Desenvolvimento da Educação Básica (IDEB) de acordo com o custo das atividades relevantes

Leandro Saraiva Dantas de Oliveira, Victor Godeiro de Medeiros Lima, Agamenon da Costa Melo

Segundo, Aneide Oliveira Araujo

custeio. Nesse sentido, qualquer um dos cinco métodos (custeio pleno, por absorção, por atividades, variável e custeio direto) pode ser adotado (Machado \& Holanda, 2010).

O Relatório Final da Comissão Interministerial de Custos (2005) estabelece que os custos são determinados pela natureza dos serviços prestados e são afetados pelo modelo de gestão específico de cada organização. Logo, tipos de organizações diferentes devem ter sistemas de custos diferentes.

\subsection{Custeio baseado em Atividades (ABC)}

As mudanças na gestão do dinheiro público passam também por mudanças no custo da gestão dos custos. Vechia (2001) afirma que o custeio baseado em atividades (Activity Based Cost - ABC)" é um método de custeio que apura o custo das atividades que integram o processo produtivo e o relaciona aos produtos ou serviços em função de direcionadores de custos". Para Raupp (2010), o custeio baseado em atividades seria de fácil aplicação para o setor público, visto que é comum a separação dos serviços públicos por atividades, por seus custos não serem consumidos por produtos, mas pelas próprias atividades.

O sistema $A B C$ atribui os custos indiretos às atividades específicas de um processo de fabricação de um produto ou serviço (Eldenburg \& Wolcott, 2007). De acordo com Garrison, Noreen e Brewer (2007), o custeio baseado em atividades difere dos métodos tradicionais pelo fato de: os custos não serem de produção; alguns custos poderem ser excluídos do produto; diversos acumuladores de custos serem utilizados; e por as taxas de atividade basearem-se no nível de atividade à capacidade plena.

O processo de implantação passa por etapas em que a literatura difere em seu número entre cinco (Garrison et al. 2007) a sete etapas (Horngren, Datar, \& Roger, 2004). Porém as principais são: identificar os objetos de custeio; identificar as atividades; atribuir custos às atividades; apurar direcionadores para cada conta de custeio; calcular e alocar custos de cada atividade ao objeto de custo (Eldenburg \& Wolcott, 2007). 
Análise do desempenho no Índice de Desenvolvimento da Educação Básica (IDEB) de acordo com o custo das atividades relevantes

Leandro Saraiva Dantas de Oliveira, Victor Godeiro de Medeiros Lima, Agamenon da Costa Melo

Segundo, Aneide Oliveira Araujo

Por não fazer divisões dos custos indiretos entre os produtos, mas pelas atividades, este método de custeio é considerado mais coerente para a determinação dos custos, contribuindo assim para aprimorar um sistema de gestão (Cunha, Haussmann, \& Grieshaber, 2009). A principal desvantagem deste método destaca-se na sua complexidade e no seu alto custo, sendo necessário frequentemente coletar, validar e registrar dados de diversas atividades e, em muitos casos, o benefício para tomada de decisão pode não compensar os dispêndios (Garrison et al. 2007).

Diante de todo o exposto, entende-se que a utilização de uma ferramenta completa como o $A B C$ em uma instituição pública de ensino é relevante pela necessidade de apropriar custo para as atividades desenvolvidas na mesma, em especial aquelas consideradas mais importantes para melhorar o desempenho e avaliar a qualidade do gasto público.

\subsection{Métodos e Abordagens Pedagógicas e Qualidade da Educação}

Costa, Akkari e Silva (2011) entrevistaram professores com o objetivo de analisar a qualidade da educação. Quando os entrevistados foram perguntados sobre quais métodos seriam mais eficientes para a melhoria da qualidade da educação, os professores responderam que uma variedade de métodos é utilizada e que não existe um método pronto ou mais eficiente, ou seja, que todos eles trazem vantagens e desvantagens. Ao perguntar sobre a colaboração família-escola, os professores comentaram que há um descaso por parte de algumas famílias, em que a educação dos filhos é delegada aos professores. Reconhece-se, portanto, a relevância dessa colaboração, sendo importante a participação dos pais junto às escolas.

Botelho, Gemignani, Madeira, Meloni, Rangel e Pioner (2014) analisaram o impacto da utilização de tecnologias de informação e comunicação na qualidade da educação dos alunos e não encontrou resultados que respaldem essa afirmação. Nicolella, Kassouf e Belluzzo (2014), ao testarem a qualificação dos docentes como ponto significativo na melhoria da aprendizagem, perceberam que a participação em 
Análise do desempenho no Índice de Desenvolvimento da Educação Básica (IDEB) de acordo com o custo das atividades relevantes

Leandro Saraiva Dantas de Oliveira, Victor Godeiro de Medeiros Lima, Agamenon da Costa Melo

Segundo, Aneide Oliveira Araujo

programas de pós-graduação contribui significativamente, porém, ao testarem para programas de atualização, não encontraram resultados significativos.

\section{PROCEDIMENTOS METODOLÓGICOS}

O presente estudo quanto aos objetivos pode ser categorizado como pesquisa descritiva, por procurar estabelecer relação entre variáveis, além de observar fatos, registrá-los, analisá-los, classificá-los e interpretá-los (Raupp \& Beuren, 2004).

Quanto aos procedimentos, foi realizado um estudo multicaso. Segundo Gil (2008), um estudo de caso caracteriza-se pelo estudo profundo e exaustivo de poucos objetos de maneira a permitir conhecimentos amplos e detalhados sobre o assunto. Raupp e Beuren (2004) afirmam que se pode executar um estudo de caso com dois ou mais sujeitos sem a necessidade de comparação entre os mesmos, constituindo um estudo multicaso.

Quanto à abordagem, o presente estudo é qualitativo por tentar explorar e entender o significado que os indivíduos ou grupo atribuem a um problema social ou humano, em que os dados são tipicamente coletados no ambiente do participante e a conclusão escrita tem uma estrutura flexível (Creswell, 2010).

Quanto à população de instituições de Ensino Fundamental, existem 62 escolas municipais na cidade de Natal. Para o estudo - como o objetivo era identificar as atividades desenvolvidas que impactaram o resultado no IDEB - houve a necessidade de selecionar as escolas que tiveram o melhor desempenho. Assim, por conveniência, três das que obtiveram os melhores desempenhos das séries iniciais, nas últimas três edições do IDEB, foram selecionadas levando em conta os seguintes critérios:

a) a que teve a maior nota na última avaliação (2011): Professor Antônio Campos e Silva;

b) a que teve a maior média nos últimos três anos (2007, 2009 e 2011): Professor Ascendino de Almeida; e 
Análise do desempenho no Índice de Desenvolvimento da Educação Básica (IDEB) de acordo com o custo das atividades relevantes Leandro Saraiva Dantas de Oliveira, Victor Godeiro de Medeiros Lima, Agamenon da Costa Melo Segundo, Aneide Oliveira Araujo

c) a que obteve o maior crescimento percentual nos exames entre 2007 e 2011: Monsenhor Joaquim Honório.

Uma vez selecionadas as instituições, foram agendadas visitas de campo para levantar informações acerca de suas funcionalidades, pois as escolas não possuíam uma gestão de custos baseada em atividades. Segundo Maher (2001), os contadores não podem implantar o sistema $A B C$ se não conhecerem bem as operações da entidade e, para identificar as atividades que direcionam custos, é necessário que participem da equipe dos diversos departamentos. Também não existe nenhuma pesquisa anterior sobre as atividades que são determinantes para um melhor desempenho no IDEB. Desse modo, foram feitas entrevistas com os professores das turmas envolvidas e os diretores das escolas no período de maio e junho de 2014, para identificar as atividades que, em suas opiniões, foram responsáveis pelo bom desempenho no IDEB, sendo este fator uma limitação desse estudo.

Os dados financeiros foram obtidos por meio da Secretaria Municipal de Educação - SME e pelas prestações de contas elaboradas pelas direções das escolas a fim de conhecer os gastos relativos a materiais e gastos dos diferentes grupos de atividades. No entanto, verificou-se, durante o trabalho, que as escolas não possuíam controle dos materiais consumidos nas diversas atividades (com exceção da atividade de simulado) e, pela impossibilidade de utilizar um método de rastreio ou rateio adequado, foi levado em consideração apenas o custo da mão de obra direta, muito em vista de ser o custo mais relevante dessas atividades.

De posse de todas essas informações, passou-se à tabulação dos dados, utilizando-se o Microsoft Excel, a fim de segregar os custos por atividade considerada relevante e demonstrar seus respectivos impactos no custo total da unidade de ensino. 
Análise do desempenho no Índice de Desenvolvimento da Educação Básica (IDEB) de acordo com o custo das atividades relevantes Leandro Saraiva Dantas de Oliveira, Victor Godeiro de Medeiros Lima, Agamenon da Costa Melo Segundo, Aneide Oliveira Araujo

\section{ANÁLISE DE RESULTADOS}

Atualmente, as escolas têm autonomia na gestão de valores recebidos por meio de transferências municipal e federal. Os recursos que chegam pela fonte municipal são oriundos do Plano de Desenvolvimento da Escola - PDE e dos Recursos do Orçamento do Município - ROM, e os recursos da fonte federal chegam por meio do Fundo Nacional de Desenvolvimento da Educação - FNDE e por programas como Atleta na Escola, Acessibilidade e Mais Educação.

Além dessas verbas recebidas por meio de transferências diretas, a SME efetua diretamente o pagamento de toda a folha dos servidores efetivos, temporários e dos terceirizados, do consumo de água, energia e telefone, bem como fornece os fardamentos dos alunos.

Apresentam-se, a seguir, as escolas nas quais foi realizado o estudo, demonstrando e discutindo seus dados financeiros, estrutura e práticas de ensino, aplicando a ferramenta de custeio escolhida.

\subsection{Escola Municipal Professor Ascendino de Almeida}

Esta escola alcançou as metas projetadas do IDEB nas últimas três avaliações, obtendo 4,8, 4,9 e 5,2 respectivamente, sendo a escola que alcançou a melhor média nos últimos 3 anos. Sua infraestrutura é composta por 13 salas de aula do $1^{\circ}$ ao $5^{\circ}$ ano, nos turnos matutino e vespertino, possuindo 661 alunos matriculados.

Os recursos recebidos por meio de transferências dos orçamentos municipais e federais seguem na Tabela 1. 
Análise do desempenho no Índice de Desenvolvimento da Educação Básica (IDEB) de acordo com o custo das atividades relevantes Leandro Saraiva Dantas de Oliveira, Victor Godeiro de Medeiros Lima, Agamenon da Costa Melo Segundo, Aneide Oliveira Araujo

Tabela 1

Valores recebidos diretamente por transferências

\begin{tabular}{|c|c|c|c|c|c|c|c|c|}
\hline \multicolumn{9}{|c|}{ Recursos Recebidos (R\$) } \\
\hline Origem & \multicolumn{2}{|c|}{ PDE } & \multicolumn{2}{|c|}{ ROM } & \multicolumn{2}{|c|}{ FNDE } & \multicolumn{2}{|c|}{ Atletana Escola } \\
\hline Natureza & Custeio & Capital & Custeio & Capital & Custeio & Capital & Custeio & Capital \\
\hline Valor & 13.300, & 5.700, & $6.114,25$ & $6.114,25$ & 9.216 & $6.144,00$ & 1.342 & 0,00 \\
\hline Subtotal & \multicolumn{2}{|c|}{$19.000,00$} & \multicolumn{2}{|c|}{$12.228,50$} & \multicolumn{2}{|c|}{$15.360,00$} & \multicolumn{2}{|c|}{$1.342,00$} \\
\hline Total Fonte & \multicolumn{4}{|c|}{$31.228,50$} & \multicolumn{4}{|c|}{$16.702,00$} \\
\hline Total Geral & \multicolumn{8}{|c|}{$47.930,50$} \\
\hline
\end{tabular}

Nota. Fonte: dados da pesquisa.

Estes recursos são recebidos para serem utilizados durante todo o ano e são gerenciados pelas próprias escolas, divididos em custeio e capital. Observa-se que $65,15 \%$ foram oriundos do orçamento municipal e $34,85 \%$ do federal, totalizando o valor de $R \$ 47.930,50$. Por sua vez, as despesas pagas diretamente pela SME constam na Tabela 2.

Tabela 2

Despesas financiadas diretamente pela SME

\begin{tabular}{l|c}
\hline \multicolumn{1}{c|}{ Descrição } & Valor (R\$) \\
\hline Professores (46) - mês & $107.370,86$ \\
\hline Outros servidores (9) - mês & $17.135,52$ \\
\hline Terceirizados (16) - mês & $27.481,76$ \\
\hline Água, Energia e Telefone (média) - mês & $3.230,00$ \\
\hline
\end{tabular}

Nota. Fonte: dados da pesquisa.

De posse dos dados financeiros, se faz necessário conhecer quais atividades foram desenvolvidas por essa escola. Segundo entrevista com os diretores e funcionários, as atividades relatadas como as mais relevantes para a obtenção da maior média nas últimas avaliações do IDEB foram: acompanhamento pedagógico semanal, recreio lúdico e simulados da Prova Brasil. 
Análise do desempenho no Índice de Desenvolvimento da Educação Básica (IDEB) de acordo com o custo das atividades relevantes

Leandro Saraiva Dantas de Oliveira, Victor Godeiro de Medeiros Lima, Agamenon da Costa Melo

Segundo, Aneide Oliveira Araujo

O Acompanhamento Pedagógico Semanal consiste em reuniões semanais com os professores de cada sala, a fim de avaliar o desempenho e cumprimento do programa. O principal objetivo desses encontros é a sistematização e o controle do fazer pedagógico.

O coordenador pedagógico e os professores de cada série passam um dia por semana discutindo as práticas pedagógicas a serem adotadas, assim como as atividades de ensino a serem desempenhadas na semana seguinte. Com esse intuito, avaliam os resultados das atividades propostas na semana anterior, buscando metodologias mais eficientes para transmissão do conteúdo e estabelecendo as metas de ensino para todas as turmas do mesmo nível, além de discutir sobre os alunos com maiores dificuldades e com necessidade de acompanhamento especial.

Esse encontro ocorre em um dia letivo comum, mas os alunos não ficam sem aula. Nesse dia, são ministradas aulas de artes, ensino religioso e educação física, que possuem professores específicos para estas disciplinas. Por exemplo: nas segundasfeiras, os professores do primeiro ano se reúnem, e os alunos de todas as turmas do primeiro ano têm aulas de ensino religioso, artes e educação física.

Os custos dessa atividade foram calculados usando-se como base os salários dos coordenadores pedagógicos e professores das disciplinas de ensino religioso, artes e educação física, visto que estes substituem os docentes no momento da realização da reunião. O valor calculado para essa atividade foi de $R \$ 10.250,78$. Considerando os 10 meses letivos, chega-se ao valor de $\mathrm{R} \$ 102.507,80$.

No Recreio Lúdico, os alunos têm um acompanhamento durante o intervalo das aulas. É comum, nas escolas, um intervalo de 30 minutos para os alunos comerem, descansarem e brincarem. Segundo a direção da referida escola, os alunos chegavam cansados e dispersos após o recreio. Então, decidiu-se controlar o intervalo.

Em todas as turmas, os professores acompanham os alunos até o refeitório e, após terem recebido a merenda escolar, os levam de volta para a sala de aula para ingerir o alimento fornecido. Isso tudo leva 10 minutos. Nos outros 20 minutos, as crianças vão para o pátio brincar. 
Análise do desempenho no Índice de Desenvolvimento da Educação Básica (IDEB) de acordo com o custo das atividades relevantes Leandro Saraiva Dantas de Oliveira, Victor Godeiro de Medeiros Lima, Agamenon da Costa Melo Segundo, Aneide Oliveira Araujo

Esse momento de brincadeira também é monitorado. Três professores por dia ficam com os alunos durante todo o intervalo para controlar as brincadeiras, evitando brigas e promovendo atividades recreativas como gincanas e atividades de aprendizado. Para esse acompanhamento, ocorre um rodízio entre os docentes, ficando 3 deles monitorando o intervalo inteiro e os demais descansam durante esses 20 minutos.

Para o cálculo dessa atividade, identificou-se o valor gasto com mão de obra direta por intervalo, ou seja, o valor gasto em um recreio com os professores, que foi de $\mathrm{R} \$ 26,92$. O cálculo é explicado conforme a Tabela 3.

Tabela 3

Memória de cálculo do gasto com professores por minuto

\begin{tabular}{l|c}
\hline Total da folha de professores & $78.270,58$ \\
\hline Total de minutos por mês de funcionamento da escola & 11.440 \\
\hline Gasto com professores por minuto & 6,84 \\
\hline Valor de 1 professor em 1 minuto - considerando 34 professores & 0,19 \\
\hline 13 professores em 10 minutos de recreio & 24,70 \\
\hline 3 professores em 20 minutos de recreio & 11,40 \\
\hline Total por Recreio Lúdico & 36,10 \\
\hline
\end{tabular}

Nota. Fonte: dados da pesquisa.

O valor total da folha de professores utilizado neste cálculo difere do total da Tabela 2 pelo fato de alguns docentes estarem alocados em atividades administrativas.

Considerando que a escola teve 200 dias letivos no ano, chega-se a um total de 400 recreios ao longo do período letivo. Portanto, o total da atividade para o ano foi de $\mathrm{R} \$ 14.440,00$.

Os Simulados foram realizados com o intuito de preparar os alunos para a Prova Brasil, pois o resultado desta é um dos itens utilizados no cálculo do desempenho no IDEB.

Segundo a direção, a maioria dos alunos não possui conhecimento do que seja um gabarito ou como funciona a prova. Então os professores das turmas que irão 
Análise do desempenho no Índice de Desenvolvimento da Educação Básica (IDEB) de acordo com o custo das atividades relevantes Leandro Saraiva Dantas de Oliveira, Victor Godeiro de Medeiros Lima, Agamenon da Costa Melo Segundo, Aneide Oliveira Araujo

prestar o exame prepararam simulados. Eles foram aplicados uma vez por semana durante os dois meses que antecederam a realização do exame.

Os custos, portanto, se devem à realização de oito simulados, em quatro turmas cada, chegando à aplicação de 32 provas ao todo. Cada simulado demorou uma hora para ser aplicado. O custo da aplicação de todos os simulados em mão de obra foi de $\mathrm{R} \$ 364,80$. Gastaram-se 5.000 folhas de papel, com o preço de $\mathrm{R} \$ 101,90$ - valor esse adquirido no orçamento fornecido pela escola. Portanto, o gasto total para essa atividade é de $R \$ 466,70$.

Tabela 4

Resumo das atividades que agregam valor na escola

\begin{tabular}{l|c|c}
\hline \multicolumn{1}{c|}{ Atividades } & R\$ & $\%$ \\
\hline Acompanhamento Pedagógico Semanal & $102.507,80$ & 5,36 \\
\hline Recreio Lúdico & $14.440,00$ & 0,75 \\
\hline Simulados da Prova Brasil & 466,70 & 0,02 \\
\hline Total & $117.414,50$ & 8,56 \\
\hline
\end{tabular}

Nota. Fonte: dados da pesquisa.

Pode-se perceber, pelos valores apresentados, que a atividade de Acompanhamento Pedagógico é a que demanda mais alocação de recursos por se configurar um trabalho contínuo de melhoramento e acompanhamento do proceder dos docentes em sala de aula, acreditando-se ser responsável por impacto considerável na Prova Brasil e também responsável por 5,36\% dos recursos da escola em um ano.

Já o Recreio Lúdico e o Simulado são atividades que, embora julgadas relevantes pelos gestores da escola ao resultado na Prova, não demandam tantos recursos, sendo estratégias interessantes para serem utilizadas por qualquer escola. Representam apenas $0,77 \%$ dos recursos totais.

\subsection{Escola Municipal Antônio Campos}

A Escola Municipal Antônio Campos obteve a maior nota na última avaliação entre as escolas públicas do município de Natal, alcançando a pontuação de 5,5. Nas 
Análise do desempenho no Índice de Desenvolvimento da Educação Básica (IDEB) de acordo com o custo das atividades relevantes Leandro Saraiva Dantas de Oliveira, Victor Godeiro de Medeiros Lima, Agamenon da Costa Melo

Segundo, Aneide Oliveira Araujo

avaliações anteriores, ela havia obtido 4,7 (em 2007) e 5,6 (em 2009). Sua infraestrutura é composta por 6 salas de aulas que são utilizadas nos turnos matutino e vespertino, tendo 339 alunos matriculados.

Os recursos recebidos por transferências, nas quais as escolas têm autonomia, seguem na Tabela 5.

Tabela 5

Valores recebidos diretamente por transferências

\begin{tabular}{c|c|c|c|c|c|c|c|c}
\hline \multicolumn{7}{c}{ Escola Professor Antônio Campos e Silva (R\$) } \\
\hline Origem & \multicolumn{2}{|c|}{ PDE } & \multicolumn{2}{c|}{ ROM } & \multicolumn{2}{c|}{ FNDE } & \multicolumn{2}{c}{ Mais Educação } \\
\hline Natureza & Custeio & Capital & Custeio & Capital & Custeio & Capital & Custeio & Capital \\
\hline Valor & $11.200,00$ & $4.800,00$ & $3.855,75$ & $3.135,75$ & $5.388,00$ & $3.592,00$ & $31.528,83$ & 0,00 \\
\hline Subtotal & $16.000,00$ & \multicolumn{2}{|c|}{$6.991,50$} & \multicolumn{2}{c|}{$8.980,00$} & \multicolumn{3}{c}{$31.528,83$} \\
\hline Total Fonte & \multicolumn{7}{c|}{$60.508,83$} \\
\hline Total Geral & \multicolumn{7}{c|}{$63.991,50$} \\
\hline
\end{tabular}

Nota. Fonte: dados da pesquisa.

Observa-se que $36,21 \%$ dos recursos se originaram do orçamento municipal e $63,79 \%$ do federal, totalizando o valor de $R \$ 63.500,33$. A maior parte dos recursos origina-se do programa Mais Educação, que proporciona atividades curriculares nos dois turnos para uma parcela dos alunos. As despesas pagas diretamente pela SME constam na Tabela 6.

Tabela 6

Despesas financiadas diretamente pela SME

\begin{tabular}{l|c}
\hline \multicolumn{1}{c|}{ Descrição } & Valor (R\$) \\
\hline Professores (29) - mês & $70.875,73$ \\
\hline Outros servidores (6) - mês & $12.530,96$ \\
\hline Terceirizados (15) - mês & $26.530,95$ \\
\hline Água, Energia e Telefone (média) - mês & $2.638,00$ \\
\hline
\end{tabular}

Nota. Fonte: dados da pesquisa. 
Análise do desempenho no Índice de Desenvolvimento da Educação Básica (IDEB) de acordo com o custo das atividades relevantes

Leandro Saraiva Dantas de Oliveira, Victor Godeiro de Medeiros Lima, Agamenon da Costa Melo

Segundo, Aneide Oliveira Araujo

Em entrevista com a diretora da escola e funcionários, consideraram-se as seguintes atividades como as mais relevantes para o bom desempenho: Clube do Livro, Carrinho Itinerante e Caderno Volante.

O Clube do Livro se dá semanalmente, com a participação de todas as turmas. Os pais dos alunos são chamados para participar da atividade, na qual todos leem um pouco: pais, alunos e professor. São 4 horas mensais destinadas a essa atividade. Todas as 12 turmas param durante uma hora, uma vez por semana, para realizar essa atividade. Dividindo-se o total gasto com professores em sala de aula em um mês $(R \$ 48.091,28)$ pelos 11.440 minutos correspondentes ao funcionamento da escola em um mês, obtém-se um custo de $\mathrm{R} \$ 4,20 / \mathrm{min}$. Logo, ao mês, considerando 4 horas de atividade ao todo, chega-se ao valor de $\mathrm{R} \$ 1.008,91$.

O Carrinho Itinerante consiste em um carrinho de supermercado com livros pedagógicos, que passa de sala em sala para que os alunos leiam com a supervisão dos professores. Para tanto, procedeu-se à mesma alocação de custos. Contabilizandose as horas de mão de obra dos professores, que seriam 4 em um mês, totaliza-se um custo de $\mathrm{R} \$ 1.008,91$.

O Caderno Volante funciona com cálculos semelhantes. A atividade consiste em um caderno que os alunos levam para casa para escrever um texto, sendo esse texto corrigido pelo professor. Por meio de entrevista com um deles, estimou-se que a média de correção de toda uma turma é de 1 hora. Logo, alocou-se o valor de $R \$ 1.008,91$ para a correção, somando-se ao valor do custo dos cadernos dos alunos, comprados pela administração do colégio. O valor dos cadernos para o ano foi de $R \$ 1.322,10$. $O$ valor total da atividade por mês é de $R \$ 1.008,91$ (mão de obra), mais $R \$ 132,21$ (cadernos em um mês), totalizando $\mathrm{R} \$ 1.141,12$. 
Análise do desempenho no Índice de Desenvolvimento da Educação Básica (IDEB) de acordo com o custo das atividades relevantes Leandro Saraiva Dantas de Oliveira, Victor Godeiro de Medeiros Lima, Agamenon da Costa Melo Segundo, Aneide Oliveira Araujo

Tabela 7

Resumo das atividades que agregam valor na escola

\begin{tabular}{l|c|c}
\hline \multicolumn{1}{c|}{ Atividades } & R\$ & $\%$ \\
\hline Clube do livro & $10.089,07$ & 0,71 \\
\hline Carrinho intinerante & $10.089,07$ & 0,71 \\
\hline Caderno volante & $11.411,18$ & 0,80 \\
\hline Total & $31.589,32$ & 2,23 \\
\hline
\end{tabular}

Nota. Fonte: dados da pesquisa.

Todas as atividades desenvolvidas pela Escola Municipal Antônio Campos são de baixo custo, porém foram consideradas relevantes pelas características de estímulo à leitura. Com esse incentivo desde cedo, os alunos aprenderiam a ler cada vez melhor com o tempo e, consequentemente, poderiam estar mais preparados para a realização de atividades avaliativas e conseguiriam melhores notas. Podem-se considerar atividades interessantes e facilmente replicáveis pelo baixo custo associado a elas, atingindo $2,23 \%$ do total dos recursos anuais escolares.

\subsection{Escola Municipal Joaquim Honório}

A escola obteve o maior crescimento percentual entre o primeiro e último exame do IDEB, saindo de 3,2 (em 2007), para 5,3 (em 2011), tendo tido um crescimento de $65,63 \%$. Ela possui 8 salas de aula nos níveis de $3^{\circ}$ ao $5^{\circ}$ ano, funcionando nos turnos matutino e vespertino, tendo 367 alunos matriculados.

Os recursos recebidos por transferências seguem na Tabela 8. 
Análise do desempenho no Índice de Desenvolvimento da Educação Básica (IDEB) de acordo com o custo das atividades relevantes Leandro Saraiva Dantas de Oliveira, Victor Godeiro de Medeiros Lima, Agamenon da Costa Melo Segundo, Aneide Oliveira Araujo

Tabela 8

Valores recebidos diretamente por transferência

\begin{tabular}{c|c|c|c|c|c|c|c|c}
\hline \multicolumn{7}{c}{ Escola Monsenhor Joaquim Honório (R\$) } \\
\hline Origem & \multicolumn{2}{|c|}{ PDE } & \multicolumn{2}{c|}{ ROM } & \multicolumn{2}{c|}{ FNDE } & \multicolumn{2}{c}{ Acessibilidade } \\
\hline Natureza & Custeio & Capital & Custeio & Capital & Custeio & Capital & Custeio & Capital \\
\hline Valor & 11.200, & 4.800, & $3.394,75$ & $3.394,75$ & 6.300, & 2.700, & 8.000, & 2.000, \\
\hline Subtotal & \multicolumn{7}{|c|}{$\mathbf{2 2 . 7 8 9 , 5 0} 6.789,50$} & \multicolumn{2}{c|}{$9.000,00$} & $10.000,00$ \\
\hline Total Fonte & \multicolumn{7}{c|}{$\mathbf{4 1 . 7 8 9 , 5 0}$} \\
\hline Total Geral & \multicolumn{7}{c|}{$\mathbf{1 9 . 0 0 0 , 0 0}$} \\
\hline
\end{tabular}

Nota. Fonte: dados da pesquisa.

Observa-se que $54,53 \%$ dos recursos foram oriundos do orçamento municipal e $45,47 \%$ do federal, totalizando o valor de $R \$ 41.789,50$. Por sua vez, as despesas pagas diretamente pela SME constam na Tabela 9.

Tabela 9

Despesas financiadas diretamente pela SME

\begin{tabular}{l|c}
\hline \multicolumn{1}{c|}{ Descrição } & Valor (R\$) \\
\hline Professores (32) - mês & $68.699,31$ \\
\hline Outros servidores (9) - mês & $18.294,40$ \\
\hline Terceirizados (16) - mês & $26.530,95$ \\
\hline Agua, Energia e Telefone (média) - mês & $9.654,00$ \\
\hline Total & $123.178,66$ \\
\hline
\end{tabular}

Nota. Fonte: dados da pesquisa.

Em entrevista realizada com a diretora da escola e com um funcionário presente, foram apontadas as seguintes atividades: Simulado da Prova Brasil, Roda de Leitura e Aula de Reforço.

Quanto ao simulado, aplicaram-se oito para cada uma das três turmas de quinto ano que o colégio possui. Portanto, 48 horas mensais foram necessárias. O total de gasto com professores em sala de aula é $\mathrm{R} \$ 61.915,92$. Cada professor custa à escola $\mathrm{R} \$ 0,18$ centavos por minuto, o que apresenta um total de $\mathrm{R} \$ 537,49$. Para a aplicação 
Análise do desempenho no Índice de Desenvolvimento da Educação Básica (IDEB) de acordo com o custo das atividades relevantes Leandro Saraiva Dantas de Oliveira, Victor Godeiro de Medeiros Lima, Agamenon da Costa Melo Segundo, Aneide Oliveira Araujo

das provas, foram utilizadas 4.000 folhas de papel, com custo total de $R \$ 81,52$. Logo, o custo total dessa atividade foi de $\mathrm{R} \$ 619,01$.

A Roda de Leitura é realizada uma vez por semana, alternando-se as turmas que participam dela. Dessa forma, ocorrem apenas 4 horas dessa atividade por mês, chegando ao valor total mensal de $\mathrm{R} \$ 44,79$.

A aula de reforço é ministrada por duas professoras do quadro da escola.

Tabela 10

Resumo das atividades que agregam valor na escola

\begin{tabular}{l|c|c}
\hline \multicolumn{1}{c|}{ Atividades } & R\$ & $\%$ \\
\hline Simulado & 619,01 & 0,04 \\
\hline Roda de Leitura & 438,00 & 0,03 \\
\hline Aula de Reforço & $47.868,90$ & 3,15 \\
\hline Total & $48.925,91$ & 3,22 \\
\hline
\end{tabular}

Nota. Fonte: dados da pesquisa.

As atividades de Simulado e Roda de Leitura são de baixo custo e, como comentado anteriormente nas outras escolas com atividades similares, são responsáveis, segundo a diretora, por parte das boas notas apresentadas e registram um total de $0,07 \%$ do total de recursos gastos.

A aula de reforço é a atividade de maior custo, pois foram alocados os salários das duas professoras responsáveis pela atividade na escola, constituindo $3,22 \%$ do total de recursos da escola. Apesar de ser um montante mais significativo, foi apontado pela escola como uma atividade de efeito considerável, pois esse acompanhamento extra possibilitaria que alunos com maiores dificuldades e dúvidas pudessem ser ajudados, contribuindo para a melhoria dos alunos da escola como um todo, alcançando, assim, melhores resultados na Prova Brasil. 
Análise do desempenho no Índice de Desenvolvimento da Educação Básica (IDEB) de acordo com o custo das atividades relevantes Leandro Saraiva Dantas de Oliveira, Victor Godeiro de Medeiros Lima, Agamenon da Costa Melo Segundo, Aneide Oliveira Araujo

\section{CONCLUSÕES}

A contabilidade de custos vem assumindo um papel importante nas entidades públicas. Os gestores têm buscado utilizar essa ferramenta para tomada de decisões, buscando tornar mais eficientes os serviços por estes prestados, diminuindo custos e eliminando ineficiências existentes.

A adoção do $A B C$ proporciona mecanismos de avaliação de resultados, permitindo a comparação entre os custos da entidade com os custos de outras entidades públicas, estimulando a melhoria de suas atividades. Assim, os resultados encontrados poderão ser utilizados como benchmarking para outras escolas com 0 objetivo de melhorar os seus desempenhos.

Com relação às atividades, observou-se pequena variação entre elas nas escolas estudadas. Percebe-se um padrão no que tange à realização de simulados e incentivo à leitura.

Observa-se que, para a realização das atividades, as escolas não precisam de recursos extras aos que já possuem. Na verdade, trata-se de uma melhor forma de gerenciamento ao alocá-los em atividades pedagógicas diferenciais. Convém registrar que, na mensuração dos custos, não foram incluídos materiais consumidos, com exceção na atividade de simulados, pelo motivo de a escola não ter mecanismos de controle sobre esses nas diversas atividades e pela impossibilidade de se utilizarem métodos de rastreio ou rateio adequados.

No entanto, espera-se contribuir para o desenvolvimento metodológico do processo de mensuração e avaliação de atividades que agregam valor para os serviços sob a responsabilidade do poder público e correspondente avaliação de desempenho.

Como recomendação para pesquisas futuras, pode-se comparar as atividades desempenhadas em escolas com baixo desempenho no IDEB, a fim de verificar se a falta de alguma atividade de fato gera impacto no resultado. Sugere-se aumentar o número de observações para analisar mais profundamente a relação entre custos e desempenho. Outra possibilidade seria estudar a viabilidade de implantação de alguma 
Análise do desempenho no Índice de Desenvolvimento da Educação Básica (IDEB) de acordo com o custo das atividades relevantes

Leandro Saraiva Dantas de Oliveira, Victor Godeiro de Medeiros Lima, Agamenon da Costa Melo

Segundo, Aneide Oliveira Araujo

atividade aqui descrita para outra instituição e acompanhar o impacto de tal ação no desempenho dos alunos.

\section{REFERÊNCIAS}

Adriano, P., Rosa, F. S. da, \& Flach., L. (2015, Outubro). Desempenho de gastos públicos no ensino fundamental nas capitais brasileiras. Congresso UFSC de Controladoria e Finanças, 6, Florianópolis, SC, Brasil.

Assessoria de Imprensa do Inep/MEC. (2011). Brasil continua a avançar. Recuperado de: <http://portal.inep.gov.br/web/portal-ideb/portal-ideb>.

Borinelli, B., Amâncio-Vieira, S. F., Negreiros, L. F. de, Pereira, R. S., \& Pereira, R. R. G. (2014). Relação entre custos, desempenho e variáveis educacionais do ensino fundamental: um estudo de caso em lbiporã-PR. Revista do Serviço Público - RSP, 65(3), 335-354.

Botelho, F., Gemignani, T., Madeira, R., Meloni, L., Rangel, M., \& Pioner, H. (2014). Sistemas de accountability nas escolas públicas brasileiras: identificando a eficácia das diferentes experiências, In: Fernandes, R., Souza, A., Botelho, F., Scorzafave, I. (orgs.). Políticas públicas educacionais e desempenho escolar dos alunos da rede pública de ensino. Ribeirão Preto: Funpec / BNDES.

Brasil. Constituição (1988). Constituição da República Federativa do Brasil. Brasília, DF: Senado Federal. Recuperado em 25 maio, 2014, de <http://www.senado.gov.br/legislacao/const/con1988/CON1988_11.02.2014/CON19 88.pdf>.

Brasil, Lei Complementar 101, de 04 de maio de 2000. Recuperado em 25 maio, 2014, de <http://www.planalto.gov.br/ccivil_03/leis/lcp/lcp101.htm>.

Brasil, Lei n. 4.320, de 17 de março de 1964. Recuperado em 25 maio, de <http://www.planalto.gov.br/ccivil_03/leis/l4320.htm>.

Brasil, Comissão Interministerial de Custos. Relatório Final, (2005). Recuperado em 25 maio, 2014, de <http://www.orcamentofederal.gov.br/biblioteca/publicacoes_tecnicas/publicacoes/R elatorio_Final_da_Comissao_de_Custos.pdf>.

Cardoso, R. L., Aquino, A. C. B., \& Bitti, E. J. da S. (2011). Reflexões para um framework da informação de custos do setor público brasileiro, Revista Administração Pública, 45(5), 1565-586. 
Análise do desempenho no Índice de Desenvolvimento da Educação Básica (IDEB) de acordo com o custo das atividades relevantes Leandro Saraiva Dantas de Oliveira, Victor Godeiro de Medeiros Lima, Agamenon da Costa Melo Segundo, Aneide Oliveira Araujo

Conselho Federal de Contabilidade. Resolução CFC № 1.129/08, NBC - T16. 2 patrimônio e sistemas contábeis. Recuperado em 20 maio, 2014, de $<$ http://www.cfc.org.br/sisweb/sre>.

Conselho Federal de Contabilidade. Resolução CFC № 1.366/11, NBC - T 16.11. Sistema de informação de custos do setor público. Recuperado em 20 maio, 2014, de <http://www.cfc.org.br/sisweb/sre>.

Creswell, J. W. (2010). Projeto de pesquisa: métodos qualitativo, quantitativo e misto. (3a ed.). Porto Alegre: Artmed.

Costa, A. S. F., Akkari, A., \& Silva, R. V. S. (2011). Educação básica no Brasil: políticas públicas e qualidade, Práxis Educacional, 7(11), 73-93.

Crozatti, J., \& Almeida, L. T. de. (2012). A qualidade do gasto público com o ensino fundamental nas escolas municipais. Encontro da ANPAD, Rio de Janeiro, RJ, Brasil, 36.

Cruvinel, D. P., \& Lima, D. V. de. (2011). Adoção do regime de competência no setor público brasileiro sob a perspectiva das Normas Brasileiras e Internacionais de Contabilidade. REPEC - Revista de Educação e Pesquisa em Contabilidade, 5, 6985.

Cunha, P. R., Haussmann, D. C. S., \& Grieshaber, C. L. (2009). Aplicação do método de custeio $\mathrm{ABC}$ em uma instituição privada de educação infantil. Revista de Contabilidade da UFBA, 3(2), 49-64.

Diniz, J. A., \& Corrar, L. J. (2011, Julho). Alocação de recursos públicos na educação fundamental: uma relação entre os gastos e desempenhos dos alunos da rede pública municipal. Congresso USP de Controladoria e Contabilidade. São Paulo, SP, Brasil, 11.

Duncombe, W., Ruggiero, J., \& Yinger J. (1996). Alternative approaches to measuring the cost of education. Holding Schools Accountable, p. 327-356.

Eldenburg, L. G., \& Wolcott, S. K. (2007). Gestão de custos: como medir, monitorar e motivar o desempenho. Rio de Janeiro: LTC.

Farenzena, N., \& Machado, M. G. F. (2006). O custo das escolas públicas municipais: resultados de uma pesquisa e implicações políticas. Revista Brasileira de Política e Administração da Educação, 22, 277-290. 
Análise do desempenho no Índice de Desenvolvimento da Educação Básica (IDEB) de acordo com o custo das atividades relevantes Leandro Saraiva Dantas de Oliveira, Victor Godeiro de Medeiros Lima, Agamenon da Costa Melo Segundo, Aneide Oliveira Araujo

Garrison, R. H., Noreen, E. W., \& Brewer, P. C. (2007). Contabilidade Gerencial. (11a ed.). Rio de Janeiro: LTC.

Gil, A. C. (2008). Como elaborar projetos de pesquisa. (6a ed.). São Paulo: Atlas.

Horngren, C. T., Datar, S. M., \& Foster, G. (2004) Contabilidade de custos: uma abordagem gerencial. São Paulo: Prentice Hall.

Machado, N., \& Holanda, V. B. de. (2010). Diretrizes e modelo conceitual de custos para o setor público a partir da experiência no governo federal do Brasil. Revista Administração Pública, Rio de Janeiro, 44(4), 791-820.

Maher, M. (2001). Contabilidade de custos: criando valor para a administração. São Paulo: Atlas.

Meyer, J. W., Ramirez, F. O., Rubinson, R., \& Boli-Bennett, J. (1977). The world educational revolution, 1950-1970. Sociology of Education, 50, 242-257.

Nicolella, A., Kassouf, A., \& Belluzzo, W. (2014). Programas de qualificação do corpo docente e sua relação com o desempenho dos estudantes, In: Fernandes, R., Souza, A., Botelho, F., \& Scorzafave, L. (orgs.). Políticas públicas educacionais e desempenho escolar dos alunos da rede pública de ensino. Ribeirão Preto: Funpec/ BNDES.

Raupp, F. M (2010). Proposta de Implementação de Custeio Baseado em Atividades no Legislativo Municipal. Pensar Contábil, Rio de Janeiro, 12(49), p. 23-30.

Raupp, F. M., \& Beuren, I. M. (2004). Metodologia da pesquisa aplicável às ciências sociais. In: Beuren, I. M. (Org.). Como elaborar trabalhos monográficos em contabilidade: teoria e prática (2a ed.). São Paulo: Atlas, p. 76-97.

Rezende, F., Cunha, A., \& Cardoso, R. L. (2010). Custos no setor público. Revista Administração Pública, Rio de Janeiro, 44(4), 789-90.

Soubbotina, T. P. (2004). Beyond economic growth: introduction to sustainable development. (2th ed.). Washington, DC: The World Bank.

Vechia, R. D. (2001). Aplicação de uma metodologia de gestão e orçamentação fundamentada no custeio baseado em atividades em uma instituição de ensino a distância. (Dissertação de Mestrado). Universidade Federal de Santa Catarina UFSC, Florianópolis, Santa Catarina, Brasil. 
Análise do desempenho no Índice de Desenvolvimento da Educação Básica (IDEB) de acordo com o custo das atividades relevantes Leandro Saraiva Dantas de Oliveira, Victor Godeiro de Medeiros Lima, Agamenon da Costa Melo Segundo, Aneide Oliveira Araujo

Data de Submissão: 25/01/2017

Data de Aceite: 06/03/2018 